\title{
Réalisme et « autre de l'art » dans les Contes de Champfleury
}

\section{Michela Lo Feudo}

\section{(2) OpenEdition}

12 Journals

Édition électronique

URL : http://journals.openedition.org/recherchestravaux/583

DOI : 10.4000/recherchestravaux.583

ISSN : 1969-6434

Éditeur

UGA Éditions/Université Grenoble Alpes

\section{Édition imprimée}

Date de publication : 15 mai 2013

Pagination : 71-90

ISBN : 978-2-84310-248-6

ISSN : 0151-1874

\section{Référence électronique}

Michela Lo Feudo, "Réalisme et « autre de l'art » dans les Contes de Champfleury », Recherches \& Travaux [En ligne], 82 | 2013, mis en ligne le 15 novembre 2014, consulté le 08 septembre 2020. URL http://journals.openedition.org/recherchestravaux/583; DOI : https://doi.org/10.4000/ recherchestravaux.583 
Michela Lo Feudo

Université Federico II, Naples

\section{Réalisme et "autre de l'art»" dans les Contes de Champfleury}

\section{$L^{\prime}$ «homme de lettres» et l'art populaire}

Pourquoi inclure l'œuvre de Champfleury (pseudonyme de Jules HussonFleury, I82I-I889) dans le cadre d'une réflexion sur les rapports entre littérature et anthropologie? Parce que cet auteur indépendant et prolifique, connu de la postérité pour son engagement dans le débat sur le réalisme des années 1850 et pour avoir encouragé les débuts artistiques de Courbet, a manifesté, au fil de toute son existence, un intérêt croissant à l'égard de la culture populaire. Sa production, vaste et hétéroclite, est caractérisée par une activité intense au sein de la "petite presse" qui cède progressivement le pas, à partir des années I850, à la publication de romans et d'études en feuilletons sur les formes d'expressions non légitimées. Sa démarche, oscillante entre journalisme militant et études historiques, entre critique littéraire, artistique et production narrative, entre pantomime et recherches musicales, témoigne de l'approche heuristique d'un auteur qui s'efforce constamment de systématiser ses réflexions autour de sujets complexes et peu explorés par ses contemporains ${ }^{\mathrm{I}}$ : son Histoire de la caricature en six volumes (I865-1880) - la première, de cette ampleur, publiée en France - ainsi que les volumes portant sur les Chansons populaires des provinces de France (I860), sur l'Histoire des faïences patriotiques sous la Révolution (1867), sur l'Histoire de l'imagerie

I. L'image d'un Champfleury chercheur qui ne cesse de revenir sur ses propres centres d'intérêt pour les développer a été au cœur du colloque organisé à Bordeaux en 2003. Cet événement - dont les actes ont été publiés en 2006 - a sans aucun doute donné une impulsion nouvelle aux études littéraires sur l'auteur. Voir G. Bonnet (dir.), Champfleury écrivain chercheur, Paris, Honoré Champion, 2006. 
populaire (1869), et sur les Vignettes romantiques (I883), recueillent régulièrement des études commencées auparavant tout en multipliant les clins d'œil à l'actualité.

L'œuvre protéiforme de cet "homme de lettres» - dans le sens vaste que cette notion avait pris au XIX ${ }^{\mathrm{e}}$ siècle - commence néanmoins à se manifester dans toute sa complexité. Dans le cadre d'une nouvelle mise en perspective critique, émerge, au premier plan, le statut bivalent d'un Champfleury à la fois écrivain et historien de l'art populaire ${ }^{2}$. Derrière une production instable apparaîtrait, de plus en plus clairement, la figure d'un auteur catalyseur de tendances esthétiques hétéroclites et de nouvelles sensibilités qui peuvent être examinées à la lumière de perspectives d'analyse récentes et d'approches transdisciplinaires inédites.

Bernard Vouilloux a montré l'originalité de la démarche de l'auteur dans son étude consacrée à un Champfleury historien des arts "mineurs" ${ }^{3}$. Son analyse, centrée sur le rapport entre texte et image dans l'ensemble des études d' "érudition " publiées à partir des années I860, présente des points de repère méthodologiques et herméneutiques intéressants pour notre propos. En premier lieu, le critique essaie de délimiter un champ de recherche dont l'unité et l'homogénéité s'avèrent problématiques, tout en rappelant qu'à l'âge de Champfleury, les formes d'expression marginalisées placent essentiellement le peuple au cœur des systèmes de production et de réception. Associées donc à des objets réalisés par le peuple et pour le peuple ${ }^{4}$, elles sont essentiellement identifiées aux artefacts qui renvoient à deux caractéristiques complémentaires : la première, d'ordre sociologique, relèverait des formes d'expression associées à l'artisanat, plutôt qu'à l'art tout court. Elles connoteraient "un mode d'expression collective», "les producteurs se fondent dans la masse anonyme du peuple et ne sauraient prétendre à la plusvalue esthétique que confere la reconnaissance d'un style individuel et que sanctionne le sceau d'une signature» ( $A S A$, p. Io). Un deuxième critère renverrait au statut médiologique des objets. Lié à son support, il engloberait, d'une part, les arts à exemplaire unique et exposés dans des espaces publics, sacrés et profanes, pourvus par conséquent d'une visibilité maximale, et les arts à fabrication sérielle ou à exemplaires multiples - tels que la poterie ou

2. Il s'agit de recherches menées surtout par des littéraires et par des spécialistes de l'image que nous évoquerons au fil de notre discours.

3. B. Vouilloux, Un art sans art: Champfleury et les arts mineurs (désormais ASA), Lyon, Fage, 2009. Dans cet ouvrage, le critique recueille et développe des recherches antérieures sur Champfleury tout en privilégiant le domaine des arts figuratifs.

4. Voir également : L. Abélès, J. Lacambre, Champfleury : l'art pour le peuple, catalogue de l'exposition réalisée au musée d'Orsay, Paris, Réunion des musées nationaux, I990. 
la gravure -, à diffusion maximale, d'autre part (ibid.). La combinaison de ces deux critères justifierait la mise en place d'un réseau composite, alimenté par des formes d'expressions différentes l'une de l'autre, telles que les faïences peintes - répertoriées par Champfleury au fil d'enquêtes menées sur le terrain des provinces de France - et la caricature, expression du comique agressif et désacralisant typique du langage journalistique parisien.

En deuxième lieu, le critique suggère que Champfleury conçoit ces études dans un rapport dynamique avec le champ culturel de l'époque. Autrement dit, sa défense et illustration des arts «mineurs» ne se limiterait pas à la pure démarche historique; elle ferait fonction de parti pris idéologique aux conséquences esthétiques sensibles, dans la mesure où Champfleury se servirait du discours sur l'art populaire pour formuler des critiques à l'égard de l'actualité littéraire. Son but implicite serait de revendiquer une poétique hostile aux nouveaux réalismes émergeant dans la seconde moitié du XIX ${ }^{\mathrm{e}}$ siècle, représentés par l'écriture artiste d'un côté, par le naturalisme de l'autre.

Si une telle démarche montre que des liens féconds existent, chez Champfleury, entre image et littérature et que ces liens se manifestent à travers une production où critique artistique et critique littéraire se croisent, il serait intéressant de développer ce questionnement en mettant en relation l'intérêt que l'écrivain a porté à l'art populaire avec sa production narrative. En particulier, si à l'époque où paraissent les recueils historiques, les romans de Champfleury sont de moins en moins nombreux - l'auteur s'éloignant progressivement des cénacles littéraires parisiens pour se consacrer aux études d'érudition et, à partir de I872, à la manufacture de Sèvres dont il est nommé conservateur - remonter aux sources des recherches de Champfleury sur l'art et la culture populaires présente des enjeux non négligeables. De la révolution de Février aux années I850, en effet, l'auteur commence à montrer une attention de plus en plus explicite à la fois à l'égard de formes d'expression atypiques et en faveur d'un projet poétique et culturel novateur plus vaste. Cette période, qui marque l'éloignement de Champfleury des milieux de la bohème littéraire et artistique - où il avait gravité avec son compagnon Henry Murger - est caractérisée par la fondation, en I850, du cénacle réaliste réuni autour du peintre Courbet et, par la suite, de Durantys. À cette époque l'écrivain, qui fréquente encore les milieux de la "petite presse", rédige des articles de critique d'art militants en même temps qu'il conçoit un nouveau

5. Champfleury fut l'un des premiers à signaler le talent de l'artiste franc-comtois. Il remarqua ses toiles au Salon de I848 et consacra à celles-ci un article dans le petit journal Le Pamphlet (28 novembre). Au Salon de l'année suivante il réalisa deux feuilletons publiés dans La Silhouette (9 articles à partir du 22 juillet) et qui furent à l'origine de relations intenses avec le peintre qui durèrent jusqu'en 1865. 
cycle littéraire : celui des Contes. Il s'agit d'un corpus composé de six volumes très variés, publiés entre I85I et I854, qui s’inscrivent dans la continuité idéologique et thématique d'une trilogie antérieure, celle des Fantaisies (I847), d'où sont extraits des textes, auxquels s'ajoutent des écrits nouveaux et des romans qui feront plus tard l'objet de publications autonomes ${ }^{6}$. Notre hypothèse est la suivante : dans le cadre d'une œuvre conçue sous l'angle de la perméabilité des genres et de l'affranchissement de tout "système ${ }^{7}$ ", les Contes font fonction de chantier stratégique pour l'élaboration à la fois des théories sur le réalisme - recueillies dans le volume-manifeste homonyme de $1857^{8}-$ et des articles sur l'art populaire, qui préparent les volumes publiés à partir de la décennie suivante.

Une telle démarche est à envisager en tenant compte des spécificités de l'écriture de Champfleury. Dans notre thèse de doctorat portant sur les rapports entre littérature et image satirique au sein de la production de l'auteur, nous avons montré que l'analyse la plus approfondie menée par celui-ci sur l'art visuel passe à travers le prisme de l'écriture créative". En déjouant constamment l'horizon d'attente d'un lectorat qui s'attendrait à des prises de position esthétiques incisives et systématiques dans des études théoriques, l'écrivain montrerait ses meilleures qualités de critique dans sa production narrative, à travers une multiplication de récits qui s'enchaînent, se répondent en écho et qui marquent, en filigrane, le développement de tendances esthétiques constamment remises en discussion.

\section{Champfleury et l'«autre de l'art». Entre littérature et anthropologie}

Or, d'un point de vue méthodologique, tout en supposant que des relations existent entre écriture littéraire et art populaire dans les Contes, on ne pourrait pas faire abstraction des enjeux anthropologiques que cette démarche

6. Il s'agit des recueils suivants : Contes : Chien-Caillou, Pauvre Trompette, Feu Miette, Paris, M. Lévy, I85I ; Contes vieux et nouveaux, Paris, M. Lévy, I852; Contes domestiques, Paris, V. Lecou, I852; Contes de printemps, Paris, V. Lecou, I853; Contes d'été, Paris, V. Lecou, I853; Contes d'automne, Paris, V. Lecou, I854. Dans les pages qui suivent, nous nous rapporterons à ce corpus auquel on ajoutera - pour des raisons que nous expliquerons plus loin - les recueils des Excentriques et des Grands Hommes du ruisseau, réunis en I852, puis en I856, en un seul volume ( $2^{\mathrm{e}}$ éd. : Slatkine reprints, 1967).

7. Ce terme récurrent sous la plume de Champfleury, renvoie à tout un échafaudage idéologique capable de rendre stériles aussi bien la vie politique et sociale que culturelle.

8. J. Champfleury, Le réalisme, Paris, M. Lévy, 1857.

9. M. Lo Feudo, Jules Champfleury (I82I-I889). Littérature et caricature, thèse de doctorat en littérature française, université Federico II de Naples en cotutelle avec l'université Paris Ouest Nanterre La Défense, soutenue en janvier 2010. 
présente, dans la mesure où nos hypothèses s'inscrivent dans un questionnement plus vaste portant sur les processus qui ont accompagné, à partir du $\mathrm{XVIII}^{\mathrm{e}}$ siècle, l'émergence et l'autonomisation de savoirs de la différence en Europe $^{\text {Io }}$. Dans le contexte français en particulier, l'œuvre de Champfleury s'inscrirait dans la continuité des transformations que l'enquête sociale a subies pendant la première moitié du XIX ${ }^{\mathrm{e}}$ siècle. À cette époque, les stratégies d'appréhension de l'altérité se multiplient : à partir de la fondation de la Société des observateurs de l'homme (I800), les modèles descriptifs relevant de disciplines telles que la statistique, l'économie et la démographie trouvent dans la polygraphie romantique un terrain d'explorations cognitive et expressive complémentaires ${ }^{\text {II }}$. D'où l'émergence d'une ethnologie du proche, pendant symétrique de l'expérience exotique, que les écrivains et les artistes auraient contribué à développer. À un moment historique où la distinction entre ethnographie et folklore n'est pas consolidée,

L'ailleurs et l'autre ne sont pas des absolus séparés, ils se déclinent selon une échelle progressive et, en Europe, comme en Amérique du Nord, l'exotisme est véritablement quotidien puisqu'il réside plus dans le regard qui le découvre que dans l'objet qui le détiendrait ${ }^{12}$.

Cette approche, ce «regard» particulier, feraient non seulement de l'analyse des nivellements sociaux une composante significative de la démarche heuristique; par un détour autoréflexif, elle favoriserait le repérage des différences internes aux savoirs impliqués au fil de l'investigation.

Or, Daniel Fabre a montré que l'étude de l'œuvre de Champfleury acquiert une importance stratégique au sein du cheminement complexe qui a déterminé la naissance de l'ethnologie en France ${ }^{\mathrm{r} 3}$. Dans son essai intitulé C'est de l'art!, le chercheur a montré que c'est au sein du champ symbolique des années I840 et, plus généralement, à l'intérieur de la révolution de la modernité, qu'il faut situer la genèse de catégories largement exploitées par les sciences humaines modernes, telles qu' "art populaire, art primitif, art des fous, art des enfants». Ces formulations, considérées à l'époque comme

Io. Cette question constitue l'un des axes de recherche développés par le LAHIC (Laboratoire d'anthropologie et d'histoire de l'institution de la culture, IIAC-EHESSCNRS) : <http://www.iiac.cnrs.fr/lahic/>.

II. Voir à ce sujet Savoirs romantiques. Une naissance de l'ethnologie, établi par D. Fabre et J.-M. Privat, Nancy, Presses universitaires de Nancy, 20 oro.

I2. Ibid., p. Iо.

I3. Nous signalons le dossier "Arts de l'enfance, enfances de l'art» de la revue Gradhiva ( $\mathrm{n}^{\circ} 9$, 2009), établi sous la direction de D. Fabre à partir du séminaire sur L'autre de l'art inauguré en 2002 à l'EHESS. Voir également : D. Fabre, "L'effet Catlin. Paris, I845-I846", Gradhiva, ${ }^{\circ}$ 3, 2006, p. 55-75; aussi surtout : "C'est de l'art! : Le peuple, le primitif, l'enfant (désormais $C D A$ )", Gradhiva, n 9, 2009, p. 4-37. 
des oxymores pour le rapprochement de notions «étrangères» l'une à l'autre, seraient à l'origine d'un geste fondamental : la reconnaissance et l'appropriation de catégories extérieures à toute hiérarchie traditionnelle dans le but de reformuler l'art à partir de ses principes constitutifs.

Dans le cadre d'un discours polyphonique, synthétisé et amplifié notamment par les réflexions philosophiques de Baudelaire, Champfleury occuperait une place intéressante et méconnue. Daniel Fabre signale en particulier cinq feuilletons sur l'art populaire, publiés par l'auteur dans le quotidien démocratique Le National de septembre à octobre I851. En s'arrêtant sur ces écrits théoriques, le chercheur montre dans quelle mesure l'expérience dépaysante des arts non légitimés offerte par l'illustration grossière à deux sous, par les chansons de province ou par les faïences décorées, produit, chez Champfleury - mais il n'est pas le seul -, un «resurgissement» des souvenirs d'enfance. Ce retour en arrière dans le temps de l'individu et à l'intérieur de la conscience artistique de l'auteur présenterait deux types de conséquences : d'une part, tout en s'inscrivant dans la quête d'une nouvelle esthétique en acte héritée du romantisme $e^{14}$, il ferait de l'art populaire - ou art "naif»", «enfantin», «sauvage», "primitif», etc. - une clé privilégiée pour renouveler principes et styles au sein de la création; d'autre part, il attribue au créateur un rôle capital, celui de saisir l'unité spatiale, temporelle, géographique et sociale dans la variété des formes d'expression adoptées par l'humanitéts.

Les discussions esthétiques alimentées par des «artistes»- le terme est envisagé dans son acception romantique - comme par exemple George Sand, Nerval, Liszt, Baudelaire, Champfleury et Flaubert, serait donc porteur de "souci[s] anthropologique $[s]^{16}$ " avant la lettre. Ceux-ci auraient contribué à inaugurer une "époque de reconnaissance d'altérités multiples mais connectées, à l'extérieur et à l'intérieur de nos mondes sociaux et aussi de nos personnes" $(C D A$, p. 6). L'expression d' 'autre de l'art» désigne alors l'émergence d'une approche renouvelée à la diversité, une approche à la fois émotive et lucide,

I4. Voir à ce sujet : J.-L. Cabanès (dir.), Romantismes, l'esthétique en acte, Paris, Presses universitaires de France, 2009.

I5. Une telle mise en perspective du rôle de Champfleury au sein du champ symbolique de son époque remet implicitement en cause l'analyse que P. Bourdieu avait menée dans Les règles de l'art. Dans cet ouvrage, l'écrivain se donne à lire comme un outsider du champ littéraire, un auteur "mineur» éclipsé par les figures dominantes des grands écrivains du XIX ${ }^{\mathrm{e}}$ siècle, tels que Baudelaire et surtout Flaubert. Le sociologue souligne en effet que ce "dominé», au sein de la pyramide sociale doué d'un faible capital culturel, n'aurait pas parcouru jusqu'au bout - avec ses camarades de la bohème, puis des cénacles réalistes - le chemin de la révolution politique et symbolique. La portée subversive de ses revendications aurait été amoindrie par une complaisance excessive à l'égard des classes défavorisées. (P. Bourdieu, Les règles de l'art. Genèse et structure du champ littéraire [1992], Paris, Seuil, I998, p. IOI)

I6. D. Fabre, «D’une ethnologie romantique», dans Savoirs romantiques, ouvr. cité, p. 8. 
qui s'inscrit dans la continuité du principe romantique - développée ensuite par Baudelaire - de la correspondance et de la complémentarité entre les arts. Avant d'aboutir à une esthétique de la modernité prônée par l'avant-garde artistique parisienne, elle connoterait «la capacité à débusquer l'art où nul ne l'aurait recherché et reconnu» (ibid., p. 9) :

[...] l'autre de l'art, c'est-à-dire toutes les formes (de tous les arts) étrangères à l'école, à l'académisme, aux normes du bon goût et aux habitudes du sens commun, devient non seulement une marge à secréter et à explorer, mais une ressource stratégique : pour les uns, qui visent à remanier les fondements mêmes de l'idée et des façons de faire de l'art, pour les autres, qui construisent des sciences de l'homme dont une part souvent implicite est vouée à comprendre les pratique et l'essence que le terme «art» désigne désormais, énigmatiquement. [...] (Ibid., p. 6)

Sous l'angle de notre cas d'étude, on peut dire que littérature et anthropologie se rencontrent, en somme, sur le terrain du champ intellectuel du romantisme et surtout du postromantisme, car c'est ici, souligne l'ethnologue, qu'on peut situer l'origine la notion de primitivisme en France (ibid., p. I2). D'un côté, l'écriture narrative se révèle en tant qu'anthropologie intuitive, outil supplémentaire (et problématique) dans la réflexion sur la culture et sur la société, capable d'alimenter une réflexion historique sur les fondements épistémologiques des sciences humaines ${ }^{17}$; de l'autre, à une époque où les codes communicatifs visuels et textuels subissent une reformulation significative et la littérature elle-même se donne à lire comme un espace métaphorique de frontière où se rencontrent, dans le contexte inédit de la presse, genres, thèmes et stratégies expressives incohérents ${ }^{18}$, analyser les textes littéraires par le biais de l'expérience dépaysante de l'altérité fait de la relation ethnographique entre soi et l'autre, entre l'autre et soi, une clé de lecture complémentaire pour creuser l'expérimentation artistique qui cache les problèmes liés à la représentation à l'âge moderne.

Si donc l'œuvre de Champfleury est fortement ancrée à cette "civilisation du journal ${ }^{19}$ » dont elle partage l'instabilité, reste donc à comprendre dans quelle mesure Champfleury s'approprie cette altérité de l'art pour l'élaboration de son projet intellectuel, les feuilletons du National ne marquant qu'une étape dans l'histoire des textes qui accompagnent la formulation d'une poétique personnelle.

I7. Je renvoie à ce propos à l'ample réflexion menée par D. Fabre et J. Jamin : «Pleine page. Quelques considérations sur les rapports entre anthropologie et littérature», L'Homme, $\mathrm{n}^{\circ}$ 203-203, 2012, 3, p. 579-6I2.

I8. M.-E. Thérenty, Mosä̈ques : être écrivain entre presse et roman (I829-I836), Paris, Honoré Champion, 2003.

19. D. Kalifa, Ph. Régnier, M.-È. Thérenty, A. Vaillant (dir.), La civilisation du journal. Histoire culturelle et littéraire de la presse française au XIX siècle, Paris, Nouveau monde, 2011. 


\section{Les Contes}

Le premier jalon de la série est inauguré en I850. Intitulé simplement Contes, il réunit l'intégralité de la trilogie des Fantaisies publiée trois années auparavant $^{20}$. À l'intérieur de ce cycle conçu stratégiquement sous le parrainage de Hugo, de Delacroix et de Balzac, Champfleury place sa production sous le signe d'une esthétique de l'hétéroclite, caractérisée par l'usage fréquent d'une intertextualité fondée sur le pastiche et la parodie, le mélange des genres, le recours à l'imagination et à la créativité, tous conçus comme moyens expressifs privilégiés. Ce corpus, qui, dans sa disparité, renvoie au régime du bric-àbrac littéraire et fait de la poétique de la fantaisie un outil de contestation et de réflexion métalittéraire, tout en remettant implicitement en cause les hiérarchies artistiques officielles ${ }^{21}$. Le passage au nouveau cycle a lieu progressivement, comme en témoigne le volume des Contes vieux et nouveaux (1852) qui est suivi d'un troisième recueil, publié la même année et intitulé Contes domestiques $^{22}$. Ce dernier comporte une préface - la première de la série - où Champfleury recadre son projet poétique. Dans ce texte, dédié à sa mère, il entend réagir aux accusations de réalisme lancées par l'opinion publique :

Tu ne trouveras dans les Contes domestiques ni parti pris ni système; si le hasard t'a mis sous les yeux des accusations de réalisme, ne t'inquiète pas de ce mot, qui est un grelot qu'on attache de force à mon cou.

Les mours de la famille, les maladies de l'esprit, la peinture du monde, les curiosités de la rue, les scènes de campagne, l'observation des passions, appartiennent également au réalisme, puisque le mot est à la mode ${ }^{23}$.

20. Il est composé des recueils : Chien-Caillou. Fantaisies d'hiver; Pauvre Trompette. Fantaisies de printemps; Feu Miette. Fantaisies d'été.

2I. Au sujet d'un thème si vaste et si important pour comprendre l'œuvre de Champfleury - et qui ne peut pas être développé dans ce contexte - voir : J.-L. Cabanès, J.-P. Saïdah (dir.), La fantaisie post-romantique, Toulouse, Presses universitaires du Mirail, 2003; J.-L. Cabanès, Le négatif. Essai sur la représentation au XIXe siècle, Paris, Garnier, $201 \mathrm{I}$.

22. Dans un article publié dans L'Ordre le 2I septembre I850 et intitulé «Mouvement des arts», Champfleury avait lancé le terme "réalisme» pour définir l'art de Courbet à partir de L'enterrement à Ornans exposé au Salon de peinture. L'année suivante s'avère une fois de plus significative pour notre propos, dans la mesure où les recherches de Champfleury sur la culture populaire, commencées avant la révolution, s’intensifient. Au début de I85I, Champfleury publie dans Le Messager de l'Assemblée une longue série d'articles portant, entre autres, sur Courbet (25-26 février) et sur Max Buchon ( $\mathrm{I}^{\text {er }}$ et 4 mars) considérés comme des «intelligences d'aujourd'hui»; deux feuilletons sont également consacrés aux chansons populaires de Gustave Mathieu et de Pierre Dupont (29 et 30 avril).

23. J. Champfleury, "À ma mère», dans Contes domestiques (désormais $C D$ ), ouvr. cité, page non foliotée. 
On peut constater que le passage du régime de la fantaisie à celui du conte réaliste a lieu dans le cadre d'un changement de "terrain" d'exploration : poético-littéraire dans le premier cas, psychologico-social dans l'autre. Cette variation de perspective s'inscrit cependant dans la continuité d'un registre de la marginalité, qui persiste : l'adoption de genres "mineurs» et d'une poétique expérimentale cède la place à un champ d'enquête "qui choque» et qui attribue à l'altérité sociale un rôle de premier plan. Si dans son texte programmatique l'écrivain mélange les thématiques et les méthodologies, ceux-ci convergent vers trois ouvrages qu'il évoque à titre d'exemple pour marquer son trajet idéologique passé et ses perspectives futures : «Le livre de Chien-Caillou, les Excentriques, les Contes domestiques», conclut Champfleury, "montrent ce que je serai, plutôt ce que je suis ${ }^{24}$.»

Le personnage de Chien-Caillou, dont le modèle est Rodolphe Bresdin (I822-I855), montre à notre sens de manière exemplaire dans quelle mesure la figure de l'artiste condense des problématiques affrontées au fil du cycle des Contes. Jeune homme talentueux mais démuni, exerçant l'art "mineur» de la gravure dans sa minuscule mansarde à Paris, Chien-Caillou est l'emblème du génie humilié par un monde moderne où l'art est dévalorisé au profit d'images de plus en plus industrialisées et reproductibles. Le passé, le présent et le futur du protagoniste sont ancrés dans une condition d'isolement déterminé par sa différence : promis par son père au métier de tanneur qu'il déteste, il s'enfuit du foyer pour se consacrer au dessin. Il rejoint alors une bande de rapins. Son génie est précoce et déterminé par la cohabitation de trois éléments cruciaux : enfance, naïveté et singularité : «Il n’avait que dix ans; il dessinait d'une façon si naïve, qu'on accrochait toutes ses œuvres dans l'atelier ${ }^{25}$.» Cependant, ce sera précisément le côté enfantin, naïf, de son talent qui provoquera l'incompréhension et la mise à l'écart de l'artiste par ses pairs : "Il songea à faire de la gravure, mais sa gravure ressemblait à ses dessins; c'était quelque chose d'allemand primitif, de gothique, de naïf et de religieux, qui donnait à rire à tout l'atelier." $(C V N$, ibid.) À cette scène de dérision collective, Champfleury oppose un moment de création solitaire où l'artiste peut donner libre cours à son inspiration :

Chien-Caillou se leva, passa son pantalon, frangé au bas comme un châle, et prit une planche de cuivre commencée. Puis il emmancha une aiguille dans un morceau de bois, burin économique, et il s'assit sur son lit.

24. Ibid.

25. J. Champfleury, Contes vieux et nouveaux (désormais CVN), ouvr. cité, p. 6 (nous soulignons). 
Pendant ce travail, la figure du graveur s'illumina d'une grimace splendide qui prouvait que son travail n'était pas tout matériel et que sa pensée passait dans son burin. Il travailla ainsi quatre heures ${ }^{26}$. (CVN, p. 7)

Cependant, une telle capacité créatrice est comme profanée par le contexte parisien des années I840 : "Chien-Caillou était de cette race de bohêmes malheureux qui restent toute leur vie bohêmes ${ }^{27}$.» Exclu par ses camarades, il aura comme unique ami un doux lapin, Petiot. Ses relations avec le père Samuel - brocanteur juif qui arrache à vil prix des œuvres du graveur et les revend en occultant la signature de l'artiste - et avec Amourette, une fille publique montrent une existence condamnée à l'anonymat qui se heurte à la marchandisation croissante des relations et des sentiments. Abandonné brusquement par sa bien-aimée, il reste seul une fois de plus. En proie à ses chagrins, il est interné peu après. Son génie a cédé la place à la folie, expression extrême de l'écart de la société.

Tout en choisissant un genre très répandu pendant le romantisme - récit de la vie d'artiste rendu populaire, par la suite, grâce aux Scènes de la vie de bohème (1845-I849) d'Henry Murger - Champfleury a tout l'air de se servir de ce registre pour le critiquer de l'intérieur. D'une part, ce récit s'inscrit dans la continuité d'une pratique fréquente à l'époque que José-Luis Diaz a analysée. Elle se caractérise par la fictionnalisation, voire la spectacularisation, de l'artiste par lui-même. Un tel dispositif, étiqueté par le critique «scénographie auctoriale", relève de la démarche d'autolégitimation des acteurs du champ culturel au sein du panorama socioculturel analysé par Bourdieu ${ }^{28}$. Dans cette perspective, l'auteur de Chien-Caillou ne cache ni sa compassion, ni son autoidentification, à l'égard de l'histoire racontée. Loin de tout principe d'impersonnalité, l'écrivain tient à souligner, dans un avis au lecteur, que : "Souvent cette histoire si gaie, si folle, si amusante, aura germé toute gonflée de larmes, de faim, de misère, dans l'esprit de celui qui l'écrira plus tard ${ }^{29}$.»

Si l'on peut donc saisir, d'une part les traces d'une quête d'autoréférences symboliques au fil du texte, émerge, d'autre part, l'exigence de dépasser

26. On constatera que des échos d'inspiration romantique reviennent dans les deux derniers extraits. Le caractère "primitif allemand", «gothique» et «religieux» de l'art de ChienCaillou est le fruit d'une expérience esthétique où le génie se manifeste dans toute sa fureur.

27. J. Champfleury, Contes, ouvr. cité, p. 20 (Contes vieux et nouveaux, ouvr. cité, p. 5-6).

28. J.-L. Diaz, L'écrivain imaginaire. Scénographies auctoriales à l'époque romantique, Paris, Honoré Champion, 2007; Devenir Balzac. Linvention de l'écrivain par lui-même, Christian Pirot, 2007. Voir également : R. Amossy et D. Maingueneau, "Autour des scénographies auctoriales : entretien avec J.-L. Diaz, auteur de L'écrivain imaginaire (2007)» [en ligne], dans Argumentation et analyse du discours, $\mathrm{n}^{\circ}$ 3, 2009. Disponible sur <http://aad.revues.org/678> [consulté le 25/03/2013].

29. J. Champfleury, Contes, ouvr. cité, p. I6 (Contes vieux et nouveaux, ouvr. cité, p. 3). 
les limites de l'autocélébration. Ce passage est explicité dans le chapitre qui oppose «les mansardes des poëtes $[s i c]$ aux mansardes réelles» (CVN, p. 8-9). Le texte est conçu selon un critère comparatif, dans la mesure où deux colonnes juxtaposées montrent, "[...] à peu près le procédé employé par les poëtes pour décrire une mansarde» (ibid., p. 8); mais cette description est accompagnée de «[...] ce que pourraient écrire les poëtes s'ils avaient l'amour de la réalité» (ibid.). L'attaque est lancée contre l'exaltation de la vie de bohème, qui cache les peines infligées à la jeunesse artiste reléguée dans des mansardes où «[l'on pourrait] trouver souvent à boire des larmes». Cette représentation trompeuse est renversée, à la fin du chapitre, par la parodie du refrain de la célèbre chanson de Béranger Le grenier (I829)" : "Dans un grenier qu'on est mal à vingt ans $s^{30}$ !»

Il s'agit, cependant, d'une démarche qui n'est pas sans risques, dans la mesure où contester l'exaltation de la vie d'artiste présente un revers de la médaille tout aussi fallacieux : l'étalage d'une vie de misère. À ce propos, Champfleury fait des capacités créatrices du graveur l'élément qui peut assurer la dignité à ce marginal de la vie moderne. Alors qu'il est condamné à l'isolement et à la pauvreté par son talent, seul le récit fait écho à ses œuvres, suggère Champfleury, et pourra le sauver de l'oubli.

\section{Sauvegarder et interroger : la double démarche de Champfleury}

Les volumes suivants des Contes développent, chacun selon des perspectives différentes, les problèmes posés par l'écrivain à travers l'histoire de ChienCaillou. En analysant l'ensemble de la série, on peut penser que Champfleury explore les formes de l'«autre de l'art» en suivant une double démarche : d'un côté, sauvegarder un art éphémère et anonyme, un patrimoine autrement condamné à la disparition; de l'autre, approfondir les spécificités de ces formes d'expression dans un questionnement plus théorique sur l'écriture de l'altérité artistique, à la croisée des perspectives esthétique et proto-ethnologique.

Une telle démarche intellectuelle fait écho à la mise en place d'une collection privée commencée vers I850 que Champfleury a enrichie toute sa vie durant : elle se composait de caricatures, d'estampes populaires, de faïences peintes, d'objets matériels qui relevaient de cultures autres et qui furent vendus aux enchères et dispersés après la mort de l'auteur. Jules Troubat a établi un

30. Une telle prise de position critique est développée dans le roman de Champfleury sur la bohème : Les Aventures de Mademoiselle Mariette, inséré à la tête du recueil des Contes de printemps (ouvr. cité) publiés en 1853 . 
parallèle intéressant entre la collection de l'auteur et les Contes domestiques. Dans Une amitié à la D'Arthez, il souligne que le roman introductif, Les Oies de Noël, est "plein du "musée des pauvres", c'est-à-dire de chansons villageoises, de complaintes et de l'enseignement que peut offrir la vieille imagerie $\mathrm{e}^{3 \mathrm{I}}$ ».

Ce recueil est, en effet, peuplé d'une imagerie hétéroclite, dans le sens vaste que Philippe Hamon a attribué au terme en analysant l'art et la littérature au $\mathrm{XIX}^{\mathrm{e}}$ siècle ${ }^{32}$ : chansons, estampes, meubles, éléments architecturaux, jeux de société, outils multiples animent un univers où les objets donnent plus d'informations sur les hommes que les personnages eux-mêmes. Si dans l'Histoire de l'imagerie populaire (I869), l'auteur avait affirmé qu' "[a]vant que l'imagerie ne disparaisse tout à fait, il faut l'étudier dans ses racines, dans sa floraison du passé, dans son essence et son développement ${ }^{33}$ ", le réseau iconique qu'il met en place dans les Contes est fondé sur un système complexe $\mathrm{d}^{\prime}$ ' objets de peu ${ }^{34}$ », souvent contextualisés, où fonction physique, artefactuelle et symbolique se fondent au sein du récit.

Les occurrences du phénomène sont multiples. Parmi elles, l'enseigne donne lieu à plusieurs reprises au fil des contes. Philippe Hamon a montré dans quelle mesure cet élément contribue à la «sursémiotisation" des espaces publics et privés de la vie au XIX ${ }^{e}$ siècle ${ }^{35}$. Support intersémiotique unissant visuel et textuel, il se caractérise par le fait de mettre la rhétorique au service de finalités commerciales. Image-seuil délimitant l'intérieur et l'extérieur, elle revient souvent sous forme d'incipit dans les romans à partir des années 1840 - d'où la célèbre enseigne du Chat qui pelote (I842) de Balzac - tout en "posant d'emblée, par la typographie d'un nom propre ou par l'image qui l'accompagne, des contenus allégoriques à cette dernière ${ }^{36}$ ".

L'histoire de Quinquet, inventeur de la lampe du même nom, montre clairement la démarche de Champfleury. Le récit débute dans le village picard de Soissons et décrit, dans un registre anecdotique, la diffusion de l'éclairage au gaz sur la voie publique. En assistant dans un café à une discussion sur les avantages et les inconvénients de ce nouveau système, Champfleury est surpris par l'affirmation d'un homme âgé qui intervient dans la discussion :

3I. J. Troubat, Une amitié à la D'Arthez: Champfleury, Courbet, Max Buchon, Paris, Lucien Duc, I900, p. 79. Les Oies de Noël fut ensuite réédité sous le titre de L'Usurier Blaizot.

32. Ph. Hamon, Imageries. Littérature et image au XIXe siècle [2003], Paris, José Corti, $2^{\mathrm{e}}$ éd. revue et augmentée, 2007.

33. J. Champfleury, Histoire de l'imagerie populaire, Paris, E. Dentu, I869, p. IX-X.

34. Sur la taxinomie relevant du statut des objets (épistémologiques et matériels) en sciences de l'homme, voir l'analyse de J.-M. Schaeffer : "Objets esthétiques?», L'Homme, $\mathrm{n}^{\circ} \mathrm{170}, 2004,2$, p. 25-45.

35. Ph. Hamon, chap. «L'image dans la ville : la rue», dans ouvr. cité, p. I47-I79.

36. Ibid., p. I5I. 
- Ah! dit en secouant la tête d'un air de regret un petit vieillard perdu dans le collet de sa redingote, du temps de Quinquet!...

Cette réticence qui contenait un hommage, une larme, une biographie, ne fut pas entendue des habitués du café. Seul, je compris le petit vieillard, et mon œil qui rencontra le sien sembla lui dire : Patience, je vengerai un jour l'inconnu de l'oubli de ses compatriotes.

QUINQUET! Ces huit lettres m'ont fait fouiller plus de trois cents volumes, avant d'avoir saisi la piste d'un renseignement sur cet inventeur ignoré. [...] Quant au quinquet, l'inventeur et l'invention étaient d'une telle modestie, qu'ils ont été étouffés par l'appareil orgueilleux et les mines hautaines de leurs sœurs, les lampes. [...] (CD, p. 298-299)

L'auteur se fixe ainsi pour objectif de reconstituer l'identité biographique et la dignité culturelle de ce personnage oublié. La figure de ce "simple ouvrier ferblantier» (ibid., p. 299) est valorisée à travers l'un de ses artefacts : Champfleury nous le montre en créateur de l'enseigne du laboratoire où il est employé, le Chasseur matinal. Il la décrit minutieusement :

- Le Chasseur matinal est une girouette très-ouvragée qui, par malheur, est empreinte du mauvais goût de l'époque. Un chasseur portant perruque en tête et fusil sous le bras, lève son œil ensommeillé sur le soleil qui pointe. C'est un chasseur vertueux! [...] Le merveilleux de cette œuvre de ferblanterie consiste dans le travail du soleil : chaque rayon est découpé à jour comme une truelle à poisson; de plus, le bras du chasseur est mobile, attachée à l'épaule par un petit clou presqu'invisible. Quand le vent souffle avec violence, il fait dresser le bras $\mathrm{du}$ chasseur vertueux vers le soleil, ce qui met au comble l'enthousiasme des Soissonnais, quoique cette girouette si artistique eût été exposée à une époque peu favorable aux œuvres d'imagination, vers la fin de la Révolution.

Indépendamment du travail sculptural, le pinceau avait ajouté ses charmes à la girouette. J'ai décrit avec le plus grand soin cette girouette, parce qu'elle rend hommage à la modestie de Quinquet. [...] seul, Quinquet avait passé des nuits à la confection de ce chef-d'œuvre. Cette enseigne donna la vogue au magasin de ferblanterie, qui dès-lors fut chargé de commandes difficiles. [...] Quinquet suffisait à tout et continuait de garder l'anonyme. ( $C D$, p. 30I)

Les qualités du personnage font de lui à la fois un artiste et un artisan. Sa maîtrise dans la combinaison et dans la manipulation des matériaux se donne à voir, non seulement dans la production d'un objet remarquable d'un point de vue technique, mais aussi, souligne Champfleury, dans la création d'une œuvre "artistique» "favorable à l'imagination ». Avant qu'apparaissent les premières histoires de l'enseigne ${ }^{37}$, Champfleury vise donc à élever l'enseigne au

37. V. Fournel, Ce quion voit dans les rues de Paris, nouv. éd. revue, corrigée et augmentée, Paris, Dentu, I867 (chap. VI : «Enseignes et affiches», p. 293-315); J.-D. Blavignac, Histoire des enseignes d'hôtelleries, d'auberges et de cabarets, Genève, Grosset, I879; É. Fournier, J. Cousin, Histoire des enseignes de Paris, Paris, Dentu, I884; J. Grand-Carteret, L'enseigne, son histoire, sa philosophie, ses particularités, les boutiques, les maisons, la rue, la réclame commerciale à Lyon, Grenoble, H. Falque et F. Perrin, 1902. 
rang de création esthétique ${ }^{38}$. Elle devient l'emblème moderne de la «noblesse d'âme» de l'artisan pauvre ${ }^{39}$.

Pour mieux souligner cet aspect du personnage, l'auteur sépare composante artistique et composante marchande de l'objet : si la réclame a attiré l'attention d'un certain nombre de clients, les profits d'une telle opération commerciale sont l'apanage du seul propriétaire de l'atelier, image typique du bourgeois avare de province. L'opposition entre peuple et bourgeoise, entre art et profit, est ultérieurement accentuée par le portrait de Quinquet, que Champfleury n'hésite pas à idéaliser : laborieux, modeste et altruiste, il en est réduit à la misère à cause de son chef jaloux, Monsieur Lardois. Ce conflit manichéen est résolu grâce à la ténacité et l'habileté de l'inventeur, qualités qui lui permettent de sortir de sa condition : c'est la découverte d'un système d'illumination novateur dont Champfleury retrace les phases d'élaboration qui pourra garantir au ferblantier sa réintégration dans la société.

Ce récit publié d'abord en feuilleton dans Le Corsaire-Satan (I847), puis recueilli dans les Contes domestiques, fait donc, encore une fois, fonction de plaidoyer a posteriori de l'artiste oublié. Champfleury fait de la fiction littéraire un terrain d'expérimentation privilégié, en illustrant dans le régime d'exemplarité fourni par le genre du conte l'un des principes qui inspireront son Histoire de l'imagerie populaire : "Ce qui touche aux enseignes, aux factures de marchands, aux saltimbanques, éclairera un jour l'histoire locale.» (HIP, p. XXIV) En particulier, la plus-value symbolique dont l'enseigne est pourvue révèle bien le statut ontologique de l'art populaire d'après Champfleury : d'un côté, elle alimente un champ épistémologique complexe et nouveau, composé d'objets matériels doués d'une dimension esthétique atypique à décrypter; de l'autre, elle leur confere une dimension documentaire digne d'attention, car ils contribuent à dévoiler une partie de l'histoire sociale et culturelle menacée de disparition.

Létude de mœurs joue donc le rôle de cadre heuristique expérimental. Dans le récit intitulé Les deux cabarets d'Auteuil, la référence à l'enseigne sert d'introduction à une enquête de ce type :

[...] j'arrivai dans la rue La Fontaine en face d'un cabaret formant l'angle de la rue; les volets gris et tristes étaient fermés et porteurs d'une affiche jaune annonçant la vente par voies notariées. Au premier étage je lus l'enseigne : À mon Désir,

38. Sur le débat suscité autour de l'enseigne pendant la seconde moitié du XIX ${ }^{\mathrm{e}}$ siècle, voir : B. Tillier, "La "peinture d'enseigne", catégorie critique et lieu commun de la modernité», Romantisme, $\mathrm{n}^{\circ} \mathrm{155}$, 2012, I, p. II-24.

39. "[L'enseigne] est, en un sens, la variante moderne, dix-neuviémiste, triviale et commerciale d'une autre image symbolique et publique, celle de l'aristocratique blason de la noblesse, qui continue d'apparaître ça et là pour caractériser tel ou tel personnage [...]» (Ph. Hamon, ouvr. cité, p. I52). 
écrit en caractères rouges. De l'autre côté de la rue, faisant face, un autre cabaret badigeonné à la chaux, est plus invitant : À mon Plaisir. Un grand orme vert protège de son ombre les tables de bois fichées en terre. Les volets verts sont ouverts à larges battants; au lieu de la triste affiche timbrée du voisin, une affiche de Bonne Bière de Mars, avec son dessin naif et ses couleurs violentes, vous met tout en joie. L'enseigne est si bien trouvée; $\grave{A}$ mon Plaisir! Tandis que l'autre $\grave{A}$ mon Désir, laisse quelque chose de vague dans l'esprit. Le lendemain, j'appris l'histoire des deux cabarets. [...] (CD, p. 225)

Champfleury saisit un ensemble de détails pittoresques pour construire un espace scénique composé de surfaces aux contrastes chromatiques nets. "Colorations bruyantes»(HIP, p. XI) et "maladresse artistique»(ibid.), typiques de l'imagerie populaire, apparaissent dans leur immédiateté. Y émerge en particulier l'affiche du cabaret $\grave{A}$ mon plaisir dont «[le] dessin naif et [1] es couleurs violentes» exposent l'observateur à une expérience esthétique inattendue qui alimente sa curiosité. Un tel événement est lié à la découverte de ces éléments triviaux, mais curieux et étonnants. Celle-ci est donc l'agent qui pousse l'écrivain à découvrir l'histoire qui se cache derrière des objets apparemment anodins. Les images grossières favorisent une démarche interprétative simple : les sensations suscitées à la vue des deux façades sont opposées - couleurs ternes et tristesse d'une part, teintes fortes et gaîté de l'autre - et annoncent un jugement de type axiologique qui sera déterminant pour l'identification des personnages, le récit étant structuré selon un schéma bipolaire qui oppose les «méchants» du cabaret À mon désir désireux d'entraver le commerce à leurs rivaux «sages» et concurrents.

Dans cet épisode où l'enseigne est conçue dans sa fonction étymologique d'enseigner, sa fonction infirmative et pédagogique consiste à dévoiler des aspects insolites de niveaux sociaux méconnus et, en même temps, à attribuer à ces mêmes réalités une esthétique intéressante. Dans Le Rhin, Victor Hugo avait déjà remarqué la nécessité d'élargir son étude de l'histoire dans une perspective anthropologique avant la lettre : "Où il n'y a pas d'église, je regarde les enseignes. Pour qui sait visiter une ville, les enseignes des boutiques ont un grand sens ${ }^{40}$." Alter ego inférieur des vestiges de jadis, elles mériteraient néanmoins, d'après Hugo, l'attention de l'écrivain, dans la mesure où elles fournissent des informations supplémentaires sur l'organisation des communautés humaines.

Si «la réclame fait [donc] du mur public une cimaise de musée ${ }^{4 \mathrm{I}}$ », dans la province des Contes l'enseigne marque les étapes d'un itinéraire fictif au sein

40. V. Hugo, «Lettre VI : Bords de la Méduse; Dinant. Namur», dans Le Rhin [I842], t. I, I858, p. 79 .

4I. Ph. Hamon, ouvr. cité, p. 20. 
d'un véritable «musée en plein vent ${ }^{42}$ », dont les objets répondent à un double questionnement que Champfleury met au cour de sa poétique : esthétique d'une part, ethnologique de l'autre. On peut lire dans cette perspective le fait qu' «il est d'usage, à Soissons, d'indiquer aux étrangers [l']enseigne [réalisée par Quinquet], dont sont aussi fiers les habitants» (CD, p. 299-300). Dans Les Oies de Noël on trouve la description suivante:

Sur la place des Orfévriers on remarque une vieille maison, plus élevée que ses voisines; au dernier étage qui forme pignon, se voit une singulière peinture à fresque, qui est d'un joyeux peintre d'enseigne ignoré. Cette fresque représente un long balcon sur lequel se promènent de jeunes souris; derrière un balustre apparait un gros chat, les prunelles pleines de feu, le corps gonflé de joie cruelle, les poils inquiets. Ce sujet, peint à la colle depuis une soixantaine d'années, s'en va tous les jours, dévoré par la pluie; il est devenu pâle et n'a plus que peu d'années à briller; malgré tout, on le cite aux voyageurs qui s'en reviennent assez désappointés d'avoir visité la Maison au Chat. (Ibid., p. I22)

Parallèlement, le projet romanesque est envisagé comme un moyen dont le collectionneur-chercheur dispose pour s'opposer à l'action destructrice du temps. S’il se donne à lire comme le chantier idéologique préparatoire au lancement d'une "archéologie nouvelle» (HIP, p. X) menée à travers les enquêtes des années I860-I880 sur l'autre de l'art, le médium littéraire, avec ses spécificités rhétoriques et poétiques, il trahit également la quête problématique d'une écriture capable de re-présenter les étapes d'observation, d'analyse et d'organisation des nouveaux objets épistémologiques que le romancier souhaite intégrer dans sa démarche intellectuelle.

Ce double aspect de la recherche de Champfleury se manifeste de manière significative lorsque l'"autre de l'art» est envisagé sur un autre "terrain», celui de la ville. Les Excentriques, par exemple, recueil qui réunit des récits publiés dans la presse de I845 à I851, montrent de manière significative comment naît l'exigence d'élaborer une poétique de l'altérité sociale et artistique dictée par la singularité des sujets intégrés dans son programme réaliste ainsi que les conflits inhérents à son propos.

Champfleury se situe, encore une fois, dans le cadre d'un genre très pratiqué à l'époque et qui, comme l'a montré Daniel Sangsue ${ }^{43}$, nait à la fin du $\mathrm{XVIII}^{\mathrm{e}}$ siècle. Ensuite, il se développe dans la première moitié du $\mathrm{XIX}^{\mathrm{e}}$ siècle à travers des textes qui tendent à remettre en question l'institution littéraire dans son ensemble et le romanesque en particulier. Le projet de Champfleury, héritier de la littérature panoramique des premières décennies du siècle, se

42. L'expression est de Balzac. Voir son Prospectus dans La Caricature morale, politique et littéraire ( $\mathrm{I}^{\mathrm{er}}$ octobre $\left.\mathrm{I} 830\right)$.

43. D. Sangsue, Le récit excentrique, Paris, José Corti, 1987. 
situe dans une sorte de "relance» de l'excentricité littéraire qui a lieu dans les années i850. Nodier, théoricien de la notion d'excentricité, avait affirmé qu' « un livre excentrique est un livre qui est fait hors de toutes les règles communes de la composition et du style, et dont il est impossible ou très difficile de deviner le but ${ }^{44}{ }^{\star}$; mais on assiste par la suite à un glissement sémantique du terme qui tend plutôt au sociologique qu'au littéraire : le récit excentrique devient un récit portant sur un excentrique, à savoir un texte qui raconte la vie et/ou les actions d'un personnage original, singulier qui, pour paraphraser Nodier lui-même, vit hors des règles communes de la société. Champfleury n'hésite pas à revendiquer son adhésion à cette tradition :

À proprement parler, ce livre n'est pas un livre, c'est une réunion d'articles que la critique a vivement reprochés à l'auteur lors de la première édition. On appuyait fortement surtout sur la bizarrerie des personnages, sur leur condition de bas étage et surtout sur le côté malsain de leur intelligence ${ }^{45}$.

Les marginaux qui défilent dans Les Excentriques relèvent des métiers de la rue, des sectes utopistes quarante-huitardes, des savants marginalisés, des artistes ratés. Si l'auteur reconnaît que ses sujets mériteraient une place «dans des livres de médecine plutôt que de littérature» $(E X, \mathrm{p}$. 2), ce recueil a tout l'air d'être un défi. Champfleury essaie de pousser son enquête littéraire plus loin, sans aucune sélection discriminante pour le choix des sujets à analyser. Cependant, il ne cache pas ses perplexités. Tout en attribuant à la fidélité du regard une place de choix, l'auteur admet qu'une écriture expérimentale, libérée de toute contrainte externe, frise l'idéalisme :

Je cherche avant tout à rendre sincèrement dans la langue la plus simple mes impressions. Ce que je vois entre dans ma tête, descend dans ma plume, et devient ce que j’ai vu. La méthode est simple, à portée de tout le monde. Mais que de temps pour se débarrasser des souvenirs, des imitations, du milieu où l'on vit, et retrouver sa propre nature ${ }^{46} !(E X$, préface)

À travers une démarche de type analogique, Champfleury vise dans un premier temps à adopter les principes de liberté et d'autonomie de la représentation pour portraiturer des individus inclassables, expression outrée de l'altérité sociale et du renversement des conventions établies. La réflexion se déplace ensuite du plan thématique aux plans interprétatif et stylistique, l'auteur montrant les limites d'une relation où - pour reprendre une fois de plus une terminologie introduite par Philippe Hamon - les images étranges soumises au regard de l'écrivain nécessitent des moyens aussi bien insolites pour devenir

44. Ch. Nodier, "Bibliographie des fous. De quelques livres excentriques», Bulletin $d u$ bibliophile, suppl. aux $\mathrm{n}^{\text {os }} 2 \mathrm{I}$ et 23, nov. I835, Paris, Techener, p. I9.

45. "Préface de la deuxième édition", dans Les Excentriques (désormais EX), ouvr. cité, p. I. 46. Nous soulignons. 
des «images à lire» recréées au sein de la page écrite. On constatera alors que le paradigme de l'«autre de l'art» est envisagé dans ce discours à travers le genre de la caricature : le recueil est en effet introduit par une dédicace à Honoré Daumier qui, sous la forme de lettre, avait été publiée dans Le Pays le 24 juillet I85I - donc quelques mois avant les feuilletons du National sur l'art populaire.

Pour sa galerie biographique, l'auteur souhaite retrouver le style du dessinateur qui avait réalisé, pour le quotidien satirique Le Charivari, la série des Bohémiens de Paris (1840-I842). D'après Champfleury, Daumier se distinguerait par sa capacité à saisir le lien profond qui unit, au sein de la physionomie de ces personnages singuliers, l'«extérieur» et l'«intérieur», leur dimension physique et psychologique, sans savoirs préconçus. Si le principe qui soutient la création est que leur «masque devient étrange» $(E X, \mathrm{p} .3)$ puisque, dit l'écrivain en citant Swedenborg, «l'homme extérieur est moulé par l'homme intérieur» (ibid.), l'auteur des Excentriques reconnaît les limites des sciences développées par Porta, par Lebrun, ou par Lavater, car ces gens singuliers présentent un régime d'incohérence, un déséquilibre entre leur esprit, leur aspect et leurs actions qui échappe au simple regard, aussi bien qu'aux approches pseudo-scientifiques. À l'égard de sujets impénétrables, l'auteur oppose ainsi la naïveté moderne d' "un homme de génie qui sache sténographier» (ibid., p. 9) aux savants et aux artistes des siècles passés : "Quelquefois ces personnages», précise l'auteur dès les premières lignes du texte, "n'ont rien de surprenant ni d'étrange dans leur costume; tout est dans leur physionomie, que leurs utopies, les rêves, les idées ont rendue bizarre.» (Ibid., p. 3) La caricature, expression d'un nouveau régime médiatique introduit par la diffusion de la presse, est donc envisagée par Champfleury comme une clé de lecture privilégiée pour interroger ces sujets énigmatiques, "bohèmes véritables, à l'esprit difficile et chagrin, souvent mystérieux comme des sphinx, et toujours indéchiffrables comme l'obélisque ${ }^{47} »$ (ibid., p. 8).

Jean-François Revel a sans doute retenu la leçon de Champfleury lorsqu'il définit la caricature une "sténographie expressive ${ }^{48}$ ». D’après celui-ci, elle se fonderait sur les procédés de la schématisation et de l'altération, grâce auxquels le dessinateur serait capable de reproduire l'image d'un individu en quelques traits parfois exagérés, parfois abrégés, voire gommés. L’habileté du caricaturiste consisterait à sélectionner et à réélaborer les détails saillants du modèle

47. Il faut toutefois préciser que l'image satirique est, aujourd'hui encore, une notion très complexe qui fait l'objet d'un nombre croissant d'études interdisciplinaires (voir notre thèse, ouvr. cité). Si à l'âge de Champfleury elle délimitait un champ sémantique nébuleux qui dépasse largement la notion de portrait (B. Vouilloux, ouvr. cité), Champfleury envisageait pour ses Excentriques surtout le sous-genre du portrait-charge. Par conséquent, dans ce contexte notre analyse se limitera à discuter le rôle joué par le portrait satirique au sein de la réflexion de l'écrivain.

48. J.-Fr. Revel, «L'invention de la caricature», L'Eil, nº I09, I964, p. 2I. 
tout en faisant osciller pertinemment la représentation entre ressemblance mimétique et manipulation expressive des signes graphiques pour obtenir des effets moqueurs. Une caricature réussie serait donc capable de révéler, en quelques simples détails physiques, plus d'informations sur la personnalité et sur le caractère de son sujet que ne pourrait le faire un portrait minutieux ${ }^{49}$.

L'auteur des Excentriques ne cache pas sa fascination pour un "genre singulier ${ }^{50} "$ qui s'affirme, à partir de la monarchie de Juillet, comme commentaire illustré de l'actualité politique, sociale et culturelle. Focalisé sur la représentation de Paris, capitale dont il vise à démasquer les contradictions et les paradoxes, il se présente comme un art populaire de la modernité dans le sens baudelairien du terme ${ }^{s I}$, un art qui s'inscrit dans le régime médiatique de la reproductibilité à grande échelle imposé par la presse industrielle tout en fondant autour de la rapidité d'exécution une de ses spécificités stylistiques.

Si fascination il y a, Champfleury n'hésitera pas à prendre du recul à l'égard d'une forme d'expression considérée à certains moments historiques comme étant trop violente, voire subversive. Dans l'Histoire de la caricature moderne, il la définira selon une formule devenue célèbre : "un art grossier, cynique, un art sans art, curieux pourtant comme expression des sentiments de révolte d'un peuple qui se réveille ${ }^{52} »$. Cependant, afin d'étudier ses marginaux de la vie moderne, l'auteur se heurte à la nécessité d'adopter une grille herméneutique pertinente. Pour explorer l'univers de ses «excentriques», il expérimente donc une approche méthodologique qu'il revendiquera ensuite dans son volume sur la caricature médiévale. Il essayera de "se faire peuple, [de] mettre son âme d'accord avec l'âme [des] siècles barbares, [de] courber la tête, [de] se faire petit avec les petits, simple avec les simples ${ }^{53}$ ». Son approche de l'art non légitimé de la caricature peut s'inscrire dans ce genre d'expérimentation qui associe des thèmes d'observation contemporains à de nouvelles pistes d'analyse.

Dans la lettre à Daumier, Champfleury compare ainsi son propre travail à celui du caricaturiste. Il établit donc un parallèle entre une expérience personnelle d'écriture et les étapes pour la réalisation d'un portrait-charge :

Je retrouve dans mes notes, si vous êtes curieux de connaître mes procédés, le premier état d'un portrait d'excentrique qui n’a pu être terminé par la malveillance de celui qui posait, par ses soupçons et par sa disparition.

49. Voir à ce sujet : G. Gurisatti, Dizionario fisiognomico, Macerata, Quodlibet, 2006.

50. L'expression est de Baudelaire : «De l'essence du rire», dans Curiosités esthétiques, éd. H. Lemaitre, Paris, Garnier, 1962, p. 24I.

5I. Je renvoie également aux essais baudelairiens «Quelques caricaturistes français» (I857) et "Quelques caricaturistes étrangers» (I857).

52. J. Champfleury, Histoire de la caricature moderne, Paris, Dentu, s. d. [I867], p. X.

53. J. Champfleury, Histoire de la caricature au Moyen Âge et sous la Renaissance, Paris, Dentu, I872, p. 67. 
C'était un homme qui tous les jours se promenait sur le Pont-Neuf, gros et gras, avec une belle figure pleine, des yeux illuminés, un peu de ventre, de longs cheveux ramenés derrière les oreilles, et dont les boucles avaient fini par graisser outrageusement le col de la redingote.

Cette belle tête bien construite, et dont les yeux fiers et noirs refoulaient les regards indiscrets des passants du Pont-Neuf, était couverte d'un chapeau que rien, excepté le crayon, ne saurait rendre. [...]

Je me laboure la tête, je grimace, je me donne beaucoup de mal pour rendre ce chapeau; je rature, je sens que je n'arriverai jamais; en ce moment je m'aperçois de l'impossibilité de la description dont nos maîtres ont cependant donné depuis vingt ans des modèles de génie ${ }^{54}$.

La présence d'un détail vestimentaire du modèle, son chapeau, est à l'origine d'une véritable crise de la représentation chez un écrivain qui doute de la possibilité de re-présenter par la parole l'image qui se présente à ses yeux. En particulier, le trait simple et rapide du caricaturiste capable de "dire plus » que la plume de l'écrivain synthétise bien le paradoxe mimétique de la caricature et ses enjeux littéraires. L'admiration pour un Daumier «qui, en quelques libres crayons, donn[e] la vie pour toujours à des êtres que les historiens futurs consulteront avec joie, pour se rendre compte de l'extérieur bourgeois de notre siècle» $(E X, \mathrm{p}$. IO), peut être l'expression d'un questionnement sur la nécessité de trouver un langage qui éclaire et légitime un lent parcours vers la création d'une archéologie du contemporain que l'auteur essaie de constituer d'abord avec ses études de mœurs, ensuite avec ses ouvrages historiques.

L'image satirique serait donc une piste privilégiée - mais non moins problématique -, utile à la constitution de paradigmes interprétatifs inédits servant à comprendre la modernité. Dans le vaste panorama fourni par les Contes, la caricature n'est pas, cependant, le seul genre envisagé. Nous avons montré ailleurs dans quelle mesure la pantomime, qui est au cœur des Contes d'automne (1854), contribue à alimenter une réflexion sur le caractère énigmatique, voire indéchiffrable, des formes d'art présentées au peuple parisien - dont font partie, aux côtés de la pantomime et de la caricature, les graffitis, les scies, etc. ${ }^{5} \mathrm{Au}$ fil de l'argumentation, la pantomime se donnerait à lire comme un langage "hiéroglyphique» actualisé, un autre univers à explorer pour comprendre les processus de réception et de création d'un art populaire moderne. L'effort de l'auteur est analogue et sa démarche ambitieuse : légitimer et définir en même temps les langages visuel et textuel de la marginalité.

54. Champfleury, «À Honoré Daumier», dans Les Excentriques, Paris, M. Lévy, I852, p. 7-8.

55. Cet article, intitulé «Égyptiens aux Funambules. Les pantomimes de Champfleury entre hiéroglyphes et arts populaires» est à paraître dans le volume de mélanges offertes à J.-L. Cabanès, établi sous la direction de P.-J. Dufief. 